\title{
Optimization of the parameters in SVM-based spectrum sensing model
}

\author{
Xuping Zhai, Tian Meng, Bingbing Yang \\ Key Laboratory of Specialty Fiber Optics and Optical Access Networks \\ Shanghai University, 200072 Shanghai, China \\ zhaixp@shu.edu.cn, molita11@163.com
}

Keywords: Spectrum Sensing, Support Vector Machine, Primary User, Radial Basis Function

\begin{abstract}
The process of spectrum sensing plays a key part in Cognitive Radio (CR). In this paper, Support Vector Machine(SVM) is applied to spectrum sensing which can judge whether the Primary User (PU) exists or not for its good performance in many fields. Data set including training set and testing set with different characteristics is generated by laboratory instruments to verify the SVM-based spectrum sensing model of good performance in actual communication environment. To solve the problem of huge training set, the paper focuses on researching the optimal parameters of the number and dimension of training set. The simulation results show that the optimal parameter of Radial Basis Function (RBF) and the size of training set and testing set are different with different characteristics of training set and testing set.
\end{abstract}

\section{Introduction}

As is known, the spectrum resources are becoming more and more scare with the rapid development of wireless communication services. In the frequency range of $0-6 \mathrm{GHz}$, the spectrum utilization rate is only $15 \%-85 \%$, the licensed spectrum have not been fully utilized[1].

To improve the spectrum utilization, cognitive radio (CR) is firstly proposed by Dr. Joseph Mitola. By seeking the spectrum hole, cognitive user (CR) can use the free frequency band of primary user (PU) to communicate. There are many traditional spectrum sensing methods, such as, energy detection[2], matched filter and cyclostationary feature detection[3].

In recent years, more and more people focus on the support vector machine which has been widely used in face detection, web pages classification, medical treatment and so on. A spectrum sensing method based on support vector machine was studied in[4]. This paper discussed the three kinds of kernel functions and the performance of spectrum sensing with Radial Basis Function (RBF) is prefer. Spectrum sensing methods based on support vector machine were researched in[5,6]. It showed that this method outperformed energy detection. The spectrum sensing based on support vector machine has many aspects that need to be studied. For example, the selection of the parameters of training set and testing set. The performance of spectrum is low in low ratio of signal to noise (SNR). In this paper, training set and testing set with different characteristics are generated by the laboratory instruments to verify the SVM-based spectrum sensing model of good performance in actual communication environment. To solve the problem of huge training set, this paper focuses on analyzing the optimal parameters like the number and dimension of training set.

\section{SVM-based spectrum sensing model}

The earliest application of SVM is linearly separable classification. Suppose the training set was $D=\left\{\left(\mathbf{x}_{1,} y_{1}\right), \ldots,\left(\mathbf{x}_{i,} y_{i}\right)\right\}, \mathbf{x}_{i} \in R^{n}, y_{i} \in\{-1,1\} ; \mathbf{x}_{i}$ represents the training set vector and $y_{i}$ denotes the class of $\mathbf{x}_{i}$. Linearly separable classification can't work when two groups of set mixed. Then the slack variables $\xi_{i}$ is added which causes little change around training set[7]. $C$ is the penalty parameter to restrict or reducing the damage degree. So the quadratic optimization record as 


$$
\begin{array}{ll}
\min _{\mathcal{W}} & \frac{1}{2}\|w\|^{2}+C \sum_{i=1}^{l} \xi_{i} \\
\text { s.t. } & y_{i}\left(w \mathbf{x}_{i}+b\right) \geq 1-\xi_{i}, \quad \xi \geq 0, \quad i=1,2, \ldots, l
\end{array}
$$

The non-linear classifier for a sample $x$ can be expressed as

$$
y=\operatorname{sgn}\left[\sum_{i=1}^{l} \alpha_{i} \mathrm{y}_{i}\left(\Phi\left(\mathbf{x}_{i}\right) \cdot \Phi(\mathbf{x})\right)+b\right] \text {. }
$$

Where $K\left(\mathbf{x}_{i}, \mathbf{x}_{j}\right)=\left(\Phi\left(\mathbf{x}_{i}\right) \cdot \Phi\left(\mathbf{x}_{j}\right)\right)$ is the kernel function. $\Phi$ is a mapping which is used to transform source set from low dimensional space to a higher dimensional space. In this paper, the kernel function is chosen as RBF for its good function in support vector machine and it is shown in Eq. 3

$$
K\left(\mathbf{x}_{i}, \mathbf{x}\right)=\exp \left(-\left\|\mathbf{x}_{i}-\mathbf{x}\right\|^{2} / 2 \sigma^{2}\right)
$$

Spectrum sensing model is actually a binary assumption model, which can classify spectrum based on the received signal. $H_{1}$ represents the presence of the primary user and cognitive user cannot access this band. On the contrary, $H_{0}$ represents its absence.

Defining $x(i)$ as the received signal samples at time $i . n(i)$ is a white gauss noise with mean zero, variance $\sigma^{2} . s(i)$ is the licensed signal to be detected. The detection model can be described as

$$
x(i)= \begin{cases}n(i), & H_{0} \\ s(i)+n(i), & H_{1}\end{cases}
$$

The receiver gets $N_{t}$ signal samples in sensing period.

$$
\boldsymbol{X}=\left[\begin{array}{llll}
x(1) & x(2) & \cdots & x\left(N_{t}\right)
\end{array}\right]
$$

Through vectorizing $\boldsymbol{X}=\left[\begin{array}{llll}x(1) & x(2) & \cdots & x\left(N_{t}\right)\end{array}\right]$, matrices $\boldsymbol{X}_{1}$ is get whose size is $M \times N \cdot N_{t}=M \times N$.

\section{Generating the training set and the testing set}

In this paper, the data set including the training set and the testing set is generated by laboratory instruments produced by R\&A including Base-band Signal Generator and Fading Simulator(AMU200A)、Vector Signal Generator(SMBV100A)、SMA、FSG·Spectrum Analyzer and RF Signal Generator(AV1442). In AMU200A the types of PU are FSK、ASK、QAM、BPSK and there are many fading scenarios like CDMA、WLAN、WIMAX and so on. The construction of the laboratory instruments is below in Fig.1.

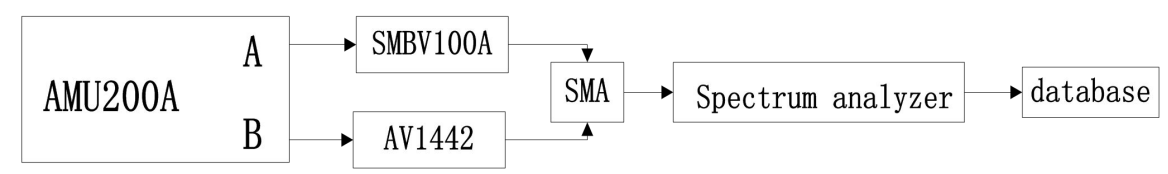

Figure 1. The construction of the laboratory instruments

AMU200A generates different amplitude base-band signal. Channel A generates Primary User, channel B generates noise. The type of PU is the signal of BPSK and the type of noise is AWGN. The bandwidth of signal is $1 \mathrm{MHz}$. The center frequency is transmitted to $2 \mathrm{GHZ}$ respectively by SMBV100A and AV1442. The sampling rate is set $10 \mathrm{MHz}$ in Spectrum Analyzer.

\section{Performance}

To find the optimal parameters of SVM spectrum sensing, the data set with different size of data set in different ratio of signal to noise (SNR) is simulated. Matlab is chosen as the simulation platform. $P_{e}$ represents the error detection probability including false detection and miss detection probability. $P_{d}$ represents right detection probability. $N$ represents the dimension of data set. 


\subsection{Optimization of the kernel function parameter}

The kernel function Radial Basis Function (RBF) has only one parameter $\sigma$. The number of training set is 200 and test set is $200 . N$ respectively equals 500 and 1000 .

Table 1. The relationship of $P e$ and $\sigma$

\begin{tabular}{|c|c|c|c|c|c|c|c|c|}
\hline$\Longrightarrow \mathrm{N}$ & \multicolumn{4}{|c|}{$\mathrm{N}=500$} & \multicolumn{4}{|c|}{$\mathrm{N}=1000$} \\
\hline$P_{e}$ & $\mathrm{SNR}=-20$ & $\mathrm{SNR}=-10$ & $\mathrm{SNR}=-5$ & $\mathrm{SNR}=0$ & $\mathrm{SNR}=-20$ & $\mathrm{SNR}=-10$ & $\mathrm{SNR}=-5$ & $\mathrm{SNR}=0$ \\
\hline 0.4 & 0.560 & 0.442 & 0.200 & 0 & 0.485 & 0.405 & 0.060 & 0 \\
\hline 0.5 & 0.554 & 0.438 & 0.190 & 0 & 0.465 & 0.395 & 0.053 & 0 \\
\hline 0.6 & 0.547 & 0.436 & 0.180 & 0 & 0.455 & 0.400 & 0.052 & 0 \\
\hline 0.7 & 0.540 & 0.435 & 0.177 & 0 & 0.455 & 0.395 & 0.051 & 0 \\
\hline 0.8 & 0.537 & 0.435 & 0.175 & 0 & 0.450 & 0.400 & 0.051 & 0 \\
\hline$\geq 0.9$ & 0.530 & 0.435 & 0.175 & 0 & 0.450 & 0.400 & 0.051 & 0 \\
\hline
\end{tabular}

SNR is respectively $-20 \mathrm{db},-10 \mathrm{db},-5 \mathrm{db}$ and $0 \mathrm{db}$. As is shown in table 1 , when $\sigma \geq 0.9, P e$ will be minimum value with $N$ in 500 and when $\sigma \geq 0.8, P e$ will be minimum value with $N$ in 1000 . Simulation researches show that optimal $\sigma$ is related to the characteristics of signal.

\subsection{The optimal number of training set}

To solve the huge training set problem, this paper is to find the optimal number of training set under good performance. The dimension of training set and testing set respectively equals 500 and 1000. SNR is from $-20 \mathrm{db}$ to $10 \mathrm{db}$ and the number of testing set is 200 .
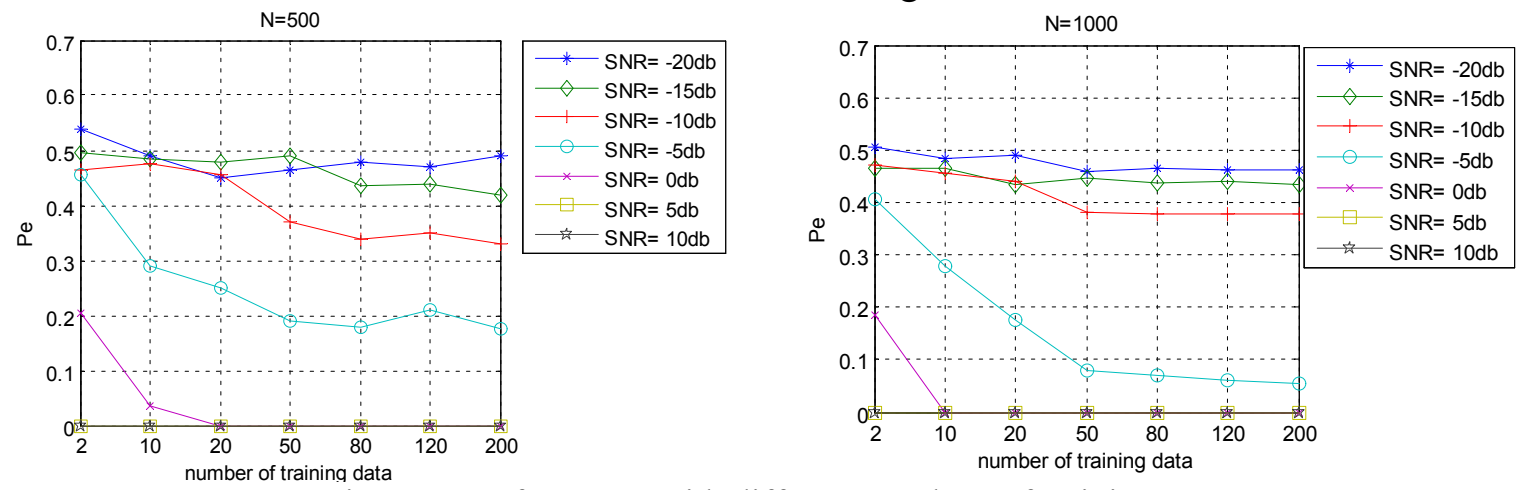

Figure 2. performance with different numbers of training set

As is shown in Fig 2, the horizontal axis representing the number of training set is from 2 to 200 . From $-5 \mathrm{db}$ to $-20 \mathrm{db}$, the performance of spectrum sensing will not become better with the number of training set larger than 80 in $\mathrm{N}=500$ and larger than 50 in $\mathrm{N}=1000$. So the number of training set can be set 80 in $\mathrm{N}=500$ and the number of training set can be set 50 in $\mathrm{N}=1000$.

\subsection{The optimal dimension of training set}

Here, the dimension of data set is from 200 to 2500 . SNR is from $-20 \mathrm{db}$ to $10 \mathrm{db}$, the number of training set 80 and testing set 200 .

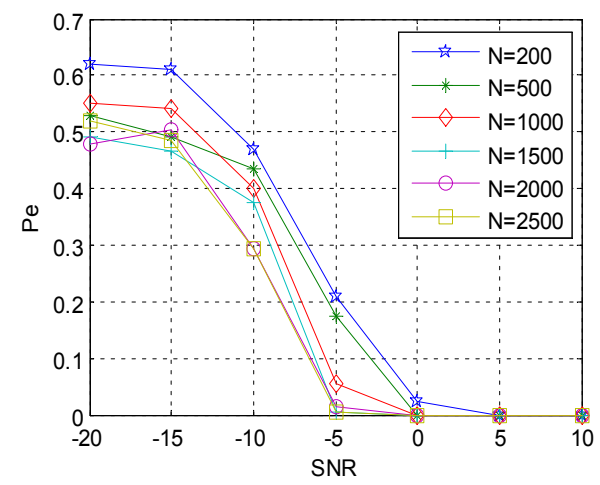

Figure 3. performance with different dimensions of data set

In Fig 3, $P_{e}$ is the lowest with $\mathrm{N}$ in 200. The dimension of training set has no much effect on the performance of spectrum sensing when the SNR is lower than $-15 \mathrm{db}$ or higher than $0 \mathrm{db}$ with $\mathrm{N}$ larger 
than 500. From $-15 \mathrm{db}$ to $-5 \mathrm{db}, P_{e}$ gets lower with the dimension becoming larger and $P_{e}$ is near to zero when the dimension is larger than $1500 \mathrm{in}-5 \mathrm{db}$. When the dimension is larger than 2000 , the performance of spectrum sensing will not become better.

\section{Conclusions}

In this paper, SVM is applied to classification problem of spectrum sensing. Firstly, it starts from SVM theory. Then, the method of getting a data set including the training set and testing set generated by laboratory instruments is described to verify the SVM-based spectrum sensing model of good performance in actual communication environment. This paper focuses on researching the optimal parameter of RBF and the number and dimension of training set. By the simulation results, it concludes that the optimal parameter of RBF and the number, dimension of training set is different with different characteristics of signals. In this paper, the received signal is without any fading. So, in the future the performance of SVM-based spectrum sensing model with data set after fading will be researched.

\section{Acknowledgments}

This work is supported by NSF China (61171085, 61401266), Innovation Program of Shanghai Municipal Education Commission (14ZZ096). Thanks Professor Wang Tao for his advices on this work.

\section{References}

[1] McHenry, Mark A. "NSF spectrum occupancy measurements project summary". Shared Spectrum Company Report, Aug. 2005.

[2] Rasheed Haroon, Rajatheva Nandana, Farah Haroon. "Spectrum Sensing with Energy Detection under Shadow-fading Condition",5th IEEE International Symposium ON Wireless Pervasive Computing (ISWPC), 2010 (5): 104 -109.

[3] Ustok R F, Ozbek B. "Spectrum sensing based on cyclostationary feature detection for cognitive radios with multiple antennas". Signal Processing and Communications Applications (SIU), 2011 IEEE 19th Conference on. IEEE, 2011: 550-553.

[4] Yang H, Xie X, Wang R. "SOM-GA-SVM Detection Based Spectrum Sensing in Cognitive Radio". Wireless Communications, Networking and Mobile Computing (WiCOM), 2012 8th International Conference on. IEEE, 2012: 1-7.

[5] Thilina K M, Choi K W, Saquib N, et al. "Pattern classification techniques for cooperative spectrum sensing in cognitive radio networks: SVM and W-KNN approaches". Global Communications Conference (GLOBECOM), 2012 IEEE. 2012: 1260-1265.

[6] X.P. Zhai, Q.M. Liu, A SVM-based spectrum sensing method, In Proc . of MCCA 2014, Oct. 2014. Xiamen, China.2014:285-289.

[7] Yuqing Huang, Hong Jiang, Hong Hu, Yuancheng Yao, "Design of Learning Engine Based on Support Vector Machine in Cognitive Radio". Computational Intelligence and Software Engineering, 2009 International Conference on. IEEE, 2009:1-4. 